\title{
Anticancer Activities and Underlying Molecular Mechanisms of Novel Mangostin Glycosides in Human Hepatocellular Carcinoma Hep3B Cells ${ }^{\dagger}$
}

\author{
Sung Min Kim, Jang Mi Han and Hye Jin Jung * \\ Department of Pharmaceutical Engineering \& Biotechnology, Sun Moon University, 70, Sunmoon-ro 221, \\ Tangjeong-myeon, Asan-si, Chungnam 336-708, Korea \\ * Correspondence:poka96@sunmoon.ac.kr \\ + Presented at the 2nd Molecules Medicinal Chemistry Symposium (MMCS): Facing Novel Challenges in \\ Drug Discovery, Barcelona, Spain, 15-17 May 2019.
}

Published: 6 August 2019

\begin{abstract}
Hepatocellular carcinoma (HCC) is the most common type of primary liver cancer and is a leading cause of cancer-related death worldwide. Therefore, exploring effective anticancer agents and their modes of action is essential for the prevention and treatment of HCC. Glycosylation can significantly improve the physicochemical and biological properties of small molecules, such as high solubility, stability increase, and lower toxicity. In this study, for the first time, we evaluated the anticancer activities of mangostin-3-O- $\beta$-D-2-deoxyglucopyranoside (Man-3DG) and mangostin 6-O- $\beta$-D-2-deoxyglucopyranoside (Man-6DG), glycosides of mangostin, against human hepatoma Hep3B cells. Our results demonstrated that Man-3DG and Man-6DG significantly suppressed growth and migration of Hep3B cells. In addition, they induced apoptosis of Hep3B cells by regulating apoptosis-related proteins of mitochondria. Noticeably, Man-3DG and Man-6DG also caused autophage, while cotreatment of the mangostin glycosides with an autophage inhibitor 3MA enhanced the inhibitory effect on Hep3B cell growth, compared to single agent treatment. Moreover, Man-3DG and Man-6DG inhibited the c-Met signaling pathway, which plays a critical role in the pathogenesis of liver cancer. Furthermore, the mangostin glycosides decreased tumor cell-induced angiogenesis in vitro through downregulation of hypoxia-inducible factor- $1 \alpha$ (HIF- $1 \alpha$ ) and vascular endothelial growth factor (VEGF). These findings suggest that Man-3DG and Man-6DG might be promising anticancer agents for HCC treatment with superior pharmacological properties than parent molecule mangostin.
\end{abstract}

Keywords: hepatocellular carcinoma; mangostin glycosides; apoptosis; autophage; c-Met signaling; tumor angiogenesis

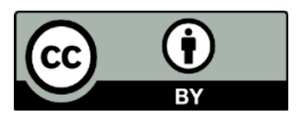

